\title{
Reconstitution des vases à reliefs monochromes d'Alaca Höyük et
} d'Eskiyapar

Néhémie Strupler

Citer ce document / Cite this document :

Strupler Néhémie. Reconstitution des vases à reliefs monochromes d'Alaca Höyük et d'Eskiyapar. In: Anatolia Antiqua, Tome 20, 2012. pp. 1-12;

doi : 10.3406/anata.2012.1320

http://www.persee.fr/doc/anata_1018-1946_2012_num_20_1_1320

Document généré le 15/05/2016 


\section{RECONSTITUTION DES VASES A RELIEFS MONOCHROMES D'ALACA HÖYÜK ET D'ESKIYYAPAR}

\section{INTRODUCTION}

$\mathrm{Au}$ Bronze récent, la céramique à reliefs est une production bien connue de la céramique hittite et offre à l'iconologuc une mine d'informations à l'instar des scènes issues du monde de la sigillographie. Animaux, personnages, mais aussi représentations architecturales appliqués en relief et rehaussés de couleurs vives animent, seuls ou organisés en scènes complexes, la paroi extérieure de certains vases, figeant ainsi une vision détaillée et vivante du monde hittite. Cette production, bien attestée depuis les fouilles d'Alişar, de Boğazköy et de Kültepe, attira immédiatement l'attention des chercheurs, mais ce n'est qu'à partir de la découverte de la jarre d'İnandiktepe, premier exemple exhumé complet ${ }^{2}$, que l'organisation des reliefs a pu être mieux comprise.

Au sein de la céramique à reliefs, certaines caractéristiques ont incité à classer les tessons en différents ensembles comme l'a fait de manière pionnière $\mathrm{K}$. Bittel ${ }^{3}$. Il forma un premier groupe défini par la polychromie qui rehausse des personnages élancés, organisés en plusieurs registres sur des lignes d'appui. Il considéra que tous les fragments découverts de ce groupe dépeignaient des processions cultuelles et les data de la période Hittite ancienne. Leur style uniforme incita à dresser un portrait archétype de cette époque : l'œil oval et fortement souligné, la pommette saillante, le nez pointu, le menton détaché et de sveltes jambes; le corps des hommes soit de profil soit de face, celui des femmes systématiquement de profil. Il s'agit de représentations stéréotypées ${ }^{4}$. Un deuxième groupe, plus lâche, réunissait les reliefs monochromes (engobés ou non) dont la production se repartissait sur tout le deuxième millénaire. Dans l'étude détaillée menée par R. M. Boehmer, quelques autres critères stylistiques furent proposés à l'aide du nouveau matériel afin d'affiner la datation ${ }^{5}$.

Les découvertes récentes à Yörüklü - Hüseyindede Tepesi et Boğazköy de vases à décors de reliefs ont apporté des données substantielles qui permettent de reconsidérer ce corpus toujours grandissant. Parmi les nouveaux vases complets découverts, on retrouve une grande jarre à quatre registres ${ }^{6}$, une petite jarre à frise unique sous le $\operatorname{col}^{7}$ et une jarre à col filiforme avec un registre sous l'épaule ${ }^{8}$. Ces trois vases ont pour dénominateur commun l'emploi de la polychromie. Ils sont datés du XVI ${ }^{\mathrm{e}}$ siècle à Yörüklü Hüseyindede Tepesi, vers 1400 à Boğazköy.

En revanche, les découvertes de céramiques monochromes sont plus rares et aucun de vase complet ne fut découvert. C'est dans ce cadre qu'il m'a semblé opportun de reprendre l'étude de deux vases à reliefs monochromes, l'un d'Alaca Höyük, l'autre d'Eskiyapar, publiés par T. Özgüç et qui, malgré leur état fragmentaire, peuvent être reconstitués dans leurs grandes lignes. Ceci permettra d'entreprendre une étude iconographique avant de s'interroger sur la place qu'ils occupent au sein de la production des vases à reliefs.

*) UMR 7044 du CNRS et de l'Université de Strasbourg ; nehemie.strupler@etu.unistra.fr.

1) Cet article est une réécriture de la conclusion du mémoire que j'ai soutenu sous la direction de Dominique Beyer à l'Université de Strasbourg. Je tiens ici à le remercier pour sa disponibilité, ses conseils et ses remarques. Un grand merci à Philippe Quenet pour ses encouragements.

2) O̊zgüç 1988

3) Bittel 1955

4) Bittel $1976: 145-146$.

5) Boehmer $1983: 21$.

6) Sipahi 2005 ; Yıldırım 2008.

7) Sipahi 2000.

8) Seeher 2007. 


\section{LE VASE D'ALACA HÖYÜK}

En 1993, alors que la céramique à reliefs provenant d'Alaca Höyük était pratiquement inconnue', T. Özgüç publia dix tessons de céramiques à reliefs ${ }^{10}$. Ceux-ci avaient été découverts en 1981 par M. Akok et, selon lui, appartiennent au niveau $3 \mathrm{~b}$ de la phase II d'Alaca Höyük. Neuf fragments, qui se distinguent par leur pâte et leur engobe, proviennent d'un même vase dont le profil est celui d'une jarre, à l'image de celles d'İnandıktepe ou de YörüklüHüseyindede Tepesi. Tout comme ces vases, les registres sont séparés par des bandes en relief qui servent de lignes d'appui aux représentations. Si l'on associe ces bandes aux différences de concavité ou de convexité des profils, on peut alors former trois groupes de tessons qui possèdent la même courbure et devaient se situer à la même hauteur (groupes de tesson A-B ; C-D ; E-H de la Fig. 1)". Ce tri opéré, il est possible de les placer verticalement, si l'on se fonde sur le profil d'une jarre. Pour la reconstruction, c'est le profil de la jarre d'İnandiktepe qui a servi de modèle. Les profils concaves ont été réunis tout en haut. Leur position est en outre assurée par la présence d'un bord du vase (A). Dans la zone médiane nous avons regroupé les profils légèrement convexes et dans la partie inférieure ceux présentant une convexité prononcée ${ }^{12}$. On reconnait in globo le thème des vases à reliefs qui associent processions de musiciens à diverses activités.

Selon cette reconstruction, il apparait distinctement que la jarre était décorée de quatre registres de reliefs et non pas d'un maximum de deux comme cela fut proposé ${ }^{13}$. De toute évidence, il est uniquement possible d'attribuer les tessons à un registre mais pas de déterminer leur ordonnance horizontale ; les fragments ne sont pas jointifs. Il est donc envisageable d'intervertir les tessons d'un même registre et de modifier ainsi la reconstitution ici proposée.

\subsection{La pâte et l'iconographie du vase}

L'engobe employé pour la confection du vase cst atypique puisqu'il s'agit d'un engobe blanc uniformément appliqué et poli sur toute la surface extérieure du vase. L'utilisation d'un engobe blanc n'est en soi pas extraordinaire ${ }^{14}$, on le retrouve d'ailleurs abondamment dans les reliefs polychromes mais plus rarement dans un emploi exclusif pour rehausser les reliefs. A Boğazköy les reliefs uniquement engobés de blanc représentent environ $10 \%$ du corpus $^{15}$. D'une façon générale, la céramique à engobe blanc devait se distinguer de la production ordinaire, comme nous le rappelle la découverte d'un vase ostentatoire à tête de taureau en ronde bosse à Boğazköy ${ }^{16}$.

Au premier registre, situé sous le col du vase, quatre personnages peuvent être restitués. Un individu imposant (1) a la tête orientée vers la gauche et le torse représenté de face avec les mains sur les hanches. Sa tête lourde, massive et large est dominée par l'œil, représenté de face. Le cou trapu, incisé, disparaît sous le col en $\mathrm{V}$ d'un vêtement décoré d'incisions de rectangles barrés d'obliques. Le personnage à sa gauche (2) porte un bonnet pointu, incisé de deux lignes horizontales ayant chacune un petit triangle incisé17. Du fragment (B) est conservé le bras d'un musicien tenant des cymbales/un tambourin (3) et la tête d'un personnage au bonnet pointu et au nez triangulaire (4).

$\mathrm{Au}$ deuxième registre seuls deux personnages sont identifiables, même si deux silhouettes ( 5 et 7 ) se devinent à la fracture de chaque côté du tesson $\mathrm{C}$. Les personnages, de profil, tiennent un objet circulaire dans la main, cymbale pour le premier (6), un miroir saisi par son manche pour le second (8). A la différence du premier registre, les habits sont décorés d'incisions circulaires faites au poinçon et un vêtement est marqué par un rectangle barré de deux diagonales. Cet élément fait penser à une poche cousue sur le vêtement. Les têtes, de modelé fruste, se distinguent

9) A Alaca Höyük, seul un tesson représentant les membres antérieurs d'un lion (?) était attribué au Bronze récent (Arık 1937 : pl. LXIX, fig. 3). Deux autres fragments d'après leur style doivent appartenir au Bronze ancien. Il s'agit d'une "idole en forme de violon" (Arık 1937 : pl. CVII, Al. b193) et d'une tête de cerf (Koşay 1951 : pl. CLXXIII, Al. 1036).

10) Özgüç 1993.

11) Le fragment I est de petite taille. Cette petitesse ne permet pas d'assurer sa position entre la II ${ }^{\text {ème }}$ et la IV ${ }^{\text {ème }}$ frise.

12) On remarque que le profil F' ne correspond pas aux autres profils ( $E^{\prime}$ et $\left.G^{\prime}-H^{\prime}\right)$ mais sa position est assurée par la présence de la double bande en relief. Dans tous les cas de figure, la forme de ce profil parait douteuse.

13) Özgüç $1993: 477$.

14) A Kuşaklı les proportions de céramiques à engobe blanc varient de 1,77\% (Mielke $2006: 38$ ) à $0,4 \%$ (Arnhold 2009:30); à Boğazköy entre $9,8 \%$ pour O.St. 3 et $0,6 \%$ pour O.St.2 (Parzinger et Sanz 1992 : 47).

15) Voir les reliefs $99,101,108,109,110,118$ et 190 de Boehmer (1983) soit 5,8\% de son corpus, 41, 46, 51, 65, 72, 77, 79, 86 de Parzinger et Sanz (1992) soit $22 \%$ de son corpus. On obtient $9,55 \%$ (15/157) si l'on cumule les données.

16) Schachner $2009 \mathrm{~b}: 36-38$.

17) T. Özgüç a identifié le bonnet comme une tiare à cornes (Özgüç 1993 : 476). 

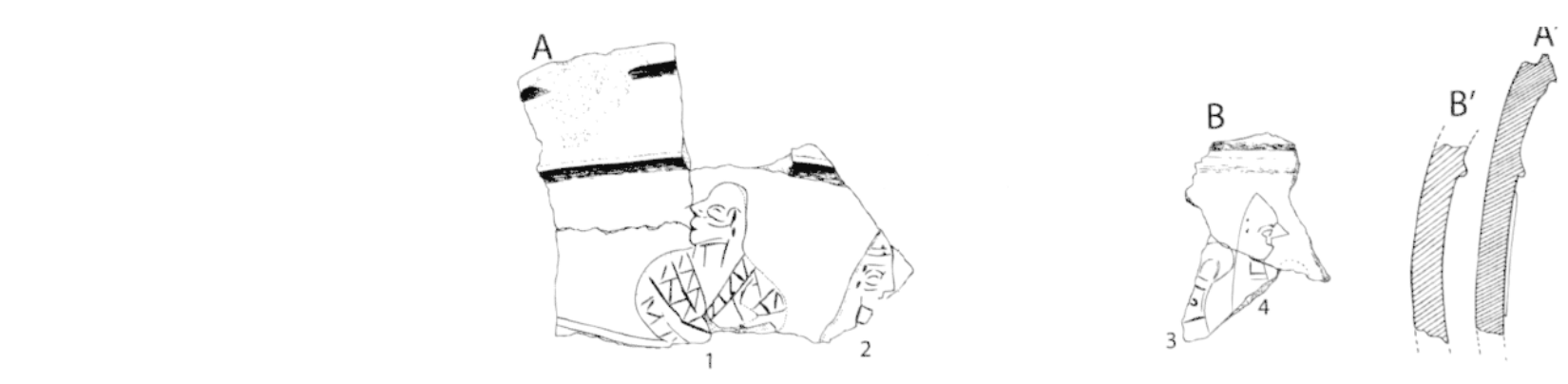

III
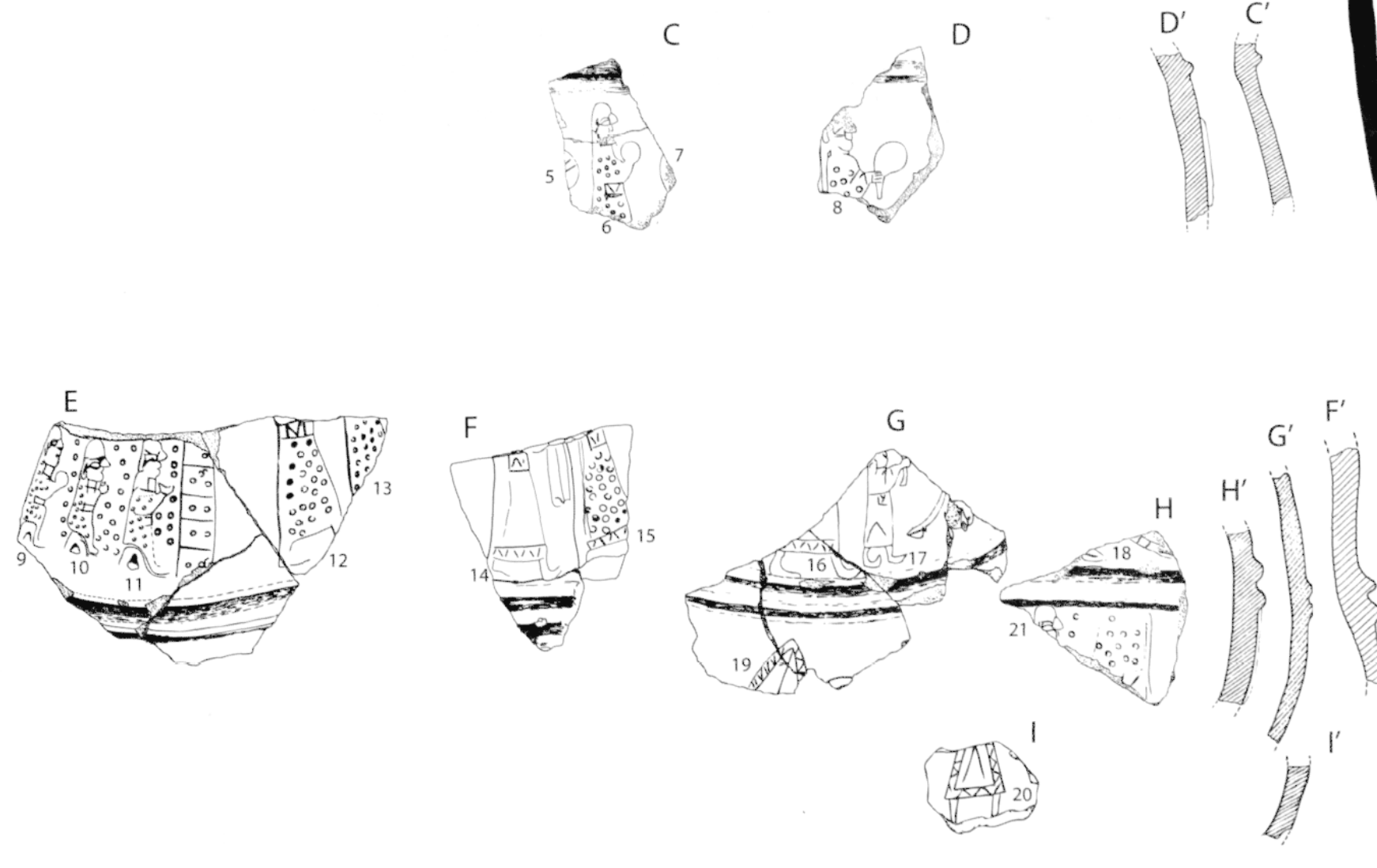

IV
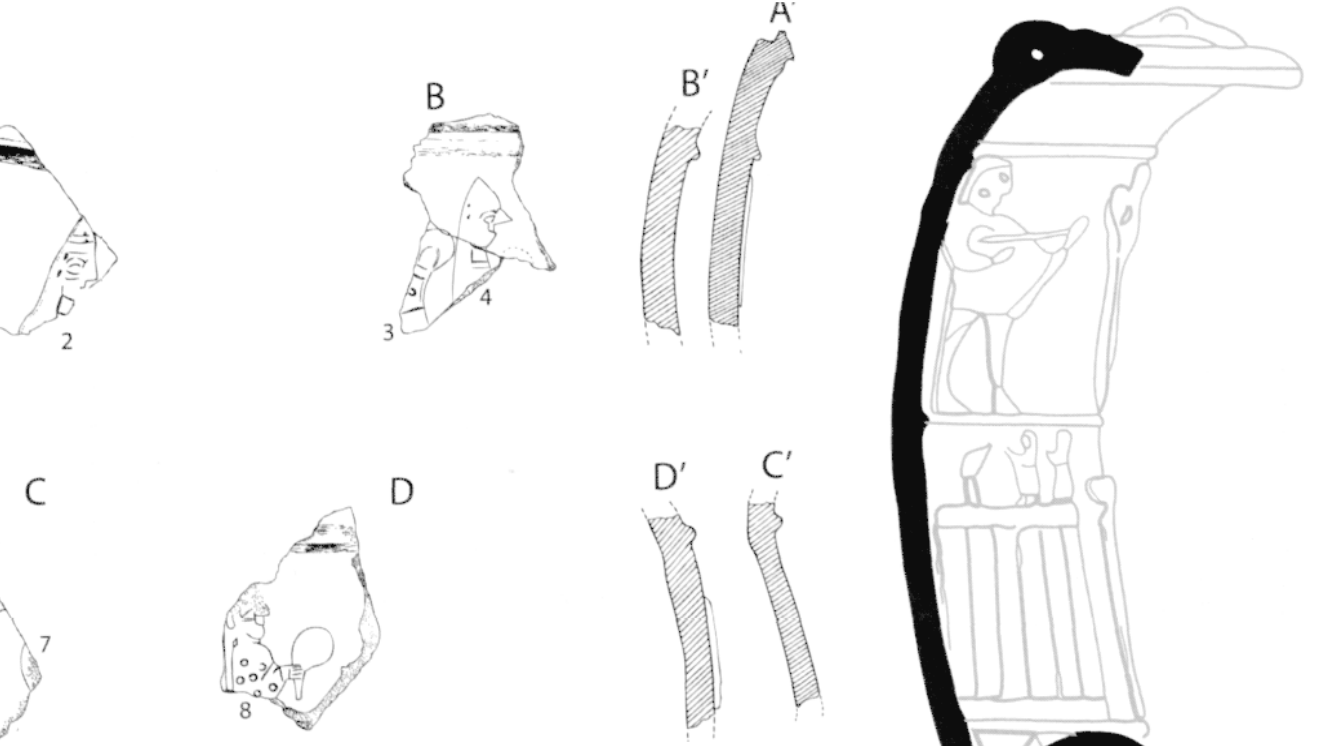
par l'absence de couvre chef; une longue mèche de cheveux court par-dessus l'épaule du personnage de droite (8). Le troisième registre, qui était le plus long, est de loin le mieux conservé. Le fragment $\mathrm{E}$ se scinde en deux représentations distinctes : sur la gauche un groupe de trois personnages assis (9-11) et sur la droite deux personnages se déplaçant (1213). Les trois individus assis sont représentés de profil, de droite, leurs vêtements et leurs postures sont identiques. Ils reposent sur un socle arrondi et évidé, les jambes tendues en avant. Les figures replient les bras en avant du buste et tiennent de gauche à droite, un objet circulaire (9) puis chacun un bol similaire (10-11). La coupe à la main fait plutôt écho à la position de prêtres (?) assis sur des sièges ${ }^{18}$. Chaque vêtement, poinçonné sur toute sa surface, est serré à la taille par une large ceinture. Les têtes sont grossièrement modelées : la joue gonflée, l'œil large en amande avec un nez considérable. Ils portent une tiare (?) ogivale qui laisse dépasser la chevelure sur la nuque. Les poinçons remplissent également le vide entre les personnages tel un horror vacui. Au devant de la scène, une bande en relief est régulièrement divisée par des incisions horizontales, délimitant des rectangles, eux-mêmes poinçonnés. Cette bande en relief rappelle les représentations d'architecture hittite, en particulier le fragment de Bitik ${ }^{19}$. Tout comme sur celui-ci, on remarque unc différence d'échelle entre les individus assis et ceux se déplaçant. Cette dichotomie insiste sur la distinction entre intérieur et extérieur, dont l'opposition est renforcée par l'horror vacui qui ne se retrouve pas au-delà de la bande en relief : les personnages sont assis à l'intérieur d'un bâtiment. Peut-on vraiment envisager de préciser la fonction du bâtiment?

A côté de cette scène, six figures (12-17) se déplacent vers la droite dans de longs vêtements similaires mais qui varient dans leur décoration. Trois robes sont poinçonnées ; deux, unies, et les trois dernières se terminent par un ourlet incisé. A l'avant, un individu de plus petite taille (17), au vêtement uni, tient la hampe d'un objet disparu. Il est précédé d'un animal ${ }^{20}$.

Le dernier registre est presque totalement perdu. On rcconnait sur le fragment $\mathrm{H}$ la tête d'un personnage au rendu grossier (21), l'oeil en amande, le nez disproportionné. Face à lui, une bande en relief verticale, décorée au poinçon, rappelle fortement la bande du tesson $\mathrm{E}$ interprétée comme un élément d'architecture. Les poinçons dans les espaces vides autour du personnage nous inclinent à reconnaître la matérialisation d'un intérieur. Le fragment I, à la position incertaine, illustre la partie inférieure d'une jupe (?) triangulaire à ourlet incisé (20). Enfin, l'élément fragmentaire du quatrième registre (19) reste énigmatique.

\subsection{Parallèles}

Les parallèles que l'on peut avancer pour comparer notre œuvre sont rares : par rapport aux autres productions le style de cette jarre est atypique. Les têtes des personnages du vase d'Alaca Höyük sont grossières, disproportionnées. D'un point de vue technique, on distingue aisément que les reliefs ont été confectionnés à la boulette ${ }^{21}$, puis achevés par le travail d'incision au couteau et au poinçon.

Les décors des vêtements, en particulier les incisions circulaires faites au poinçon, se retrouvent sur d'autres exemples connus : le vêtement d'un personnage monochrome d'Alişar (Fig. 2) ${ }^{22}$; la ceinture, le corps et la bourse d'un personnage monochrome de Boğazköy (Fig. 3$)^{23}$; et sporadiquement sur les animaux à Boğazköy ${ }^{24}$ ou à Eskiyapar ${ }^{25}$.

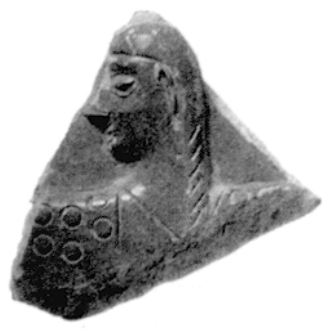

Fig. 2 : Fragment provenant d'Alaca Höyük (von der Osten 1937 : pl. I, fig. 3).

18) Voir les personnages 6 et 31 du vase d'İnandıktepe (Özgüç $1988: 175$, fig. 64). T. Özgüç reconnait des dieux-montagnes dans les personnages assis. Nous nous opposons à cette hypothèse car, bien que les supports évidés puissent évoquer des montagnes (voir par exemple Beyer 2001 : 336-337), les dieux-montagnes ne $s$ 'assoient pas sur les montagnes.

19) Ózgüç 1957.

20) Le fragment $\mathrm{H}$ a été placé devant le fragment $\mathrm{G}$ car ce dernier illustre les pattes d'un animal (18). T. Özgüç avait déjà reconnu cette similitude, mais avait préféré placer les tessons sur deux registres différents, même s'il reconnaissait pour les deux fragments la représentation d'un cerf (Özgüç $1993: 481$ ). Il est hasardeux de déterminer la nature de l'animal entre cervidés, caprinés, bovinés ou encore sphinx : comparer avec la note 44.

21) Sur cette technique, voir d'une manière générale Baudry $1978: 60$. Il s'agit d'un travail "par addition progressive de boulettes soudées entre elles à l'ébauchoir ou à la spatule" tandis que "les creux sont obtenus en retranchant de la matière à l'aide de mirettes".

22) von der Osten $1937:$ pl. I, fig. $3=$ Bittel $1976: 143$, fig. 141.

23) Photographie dans Neve $1992: 31$, fig. 78 et dessin dans Parzinger et Sanz 1992 : pl. 67, fig. 62.

24) Sur l'épaule d'un quadrupède (Parzinger et Sanz $1992:$ pl. 66, fig. 47) et sur un bovidé (Boehmer 1983).

25) Sur le corps des bovidés (Özgüç 1982). 


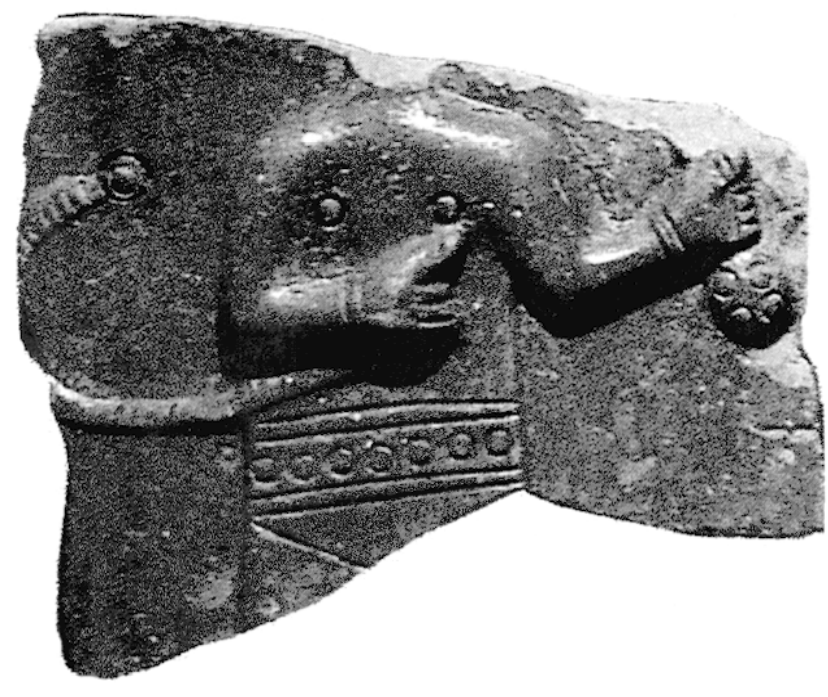

Fig. 3 : Fragment provenant de Boğazköy (Neve $1992:$ 31, fig. 78c).

Bien que ces parallèles soient de styles différents, puisqu'ils s'inscrivent dans la lignée des représentations stéréotypées de la céramique à reliefs, ils sont tous monochromes et recouverts d'un engobe épais, rouge ou brun.

Pour la céramique à reliefs, l'utilisation d'incisions est également peu répandue étant donné que, là encore, la majorité des reliefs étaient mis en valeur par l'utilisation de couleurs bigarrées. A nouveau, ce sont les tessons monochromes qui forment les meilleurs parallèles : le tesson monochrome rouge, déjà cité et découvert à Boğazköy (Fig. 3), d'un personnage décoré de poinçons où les incisions soulignent la bordure de la ceinture, l'extrémité des manches et l'articulation de la hanche ${ }^{26}$; l'ourlet des figures frontales entourant un col filiforme d'une jarre à Alişar ${ }^{27}$; un personnage monochrome blanc sur le col d'une bouteille de Boğazköy ${ }^{28}$; le long vêtement incisé d'un bandeau de chevrons à l'arrière d'un personnage d'un vase de Boğazköy ${ }^{29}$.

En ce qui concerne les lypes de vêtements, $T$. Özgüç avait proposé une classification à partir du vase d'Inandiktepe ${ }^{30}$, mais les ornements de ceux-ci sont peints et de style différent ce qui ne permet pas d'établir de comparaisons. Seul le vêtement de l'acrobate 58 d'İnandıktepe ${ }^{31}$, dont le col et les manches sont peints de traits obliques, offre un bon pendant au personnage 1 .

\subsection{Ordonnance au sein de la production}

Quelles conclusions tirer des rapprochements fournis ici ? Tout d'abord, tant du point de vue du style que de la facture, le vase à quatre registres d'Alaca Höyük se distingue de la majorité des reliefs qui appartiennent au groupe polychrome. Les meilleurs parallèles proviennent du corpus monochrome, même si au sein de cette production la couleur de prédilection de l'engobe semble avoir été le rouge-brun. Les vêtements n'offrent pas de point de comparaison à ce qui est connu et le style des personnages est sans pendant. Seule l'utilisation du poinçon et du couteau, rclcvéc ici ou là, permet d'établir quelques comparaisons. Cette facture atypique soulève la question d'un atelier, mais la répartition étendue des fragments comparables (Boğazköy, Eskiyapar, Alişar et Alaca Höyük) ne permet pas de conclure. En contrepartie, il faut souligner les éléments qui rattachent le vase à la production de céramique à reliefs : sa forme de jarre, la division en quatre frises à reliefs marquée par des lignes d'appui, la dichotomie intérieur/extérieur, l'aspect général de la représentation alliant musiciens, animaux et cortège de prêtres (?). Nous sommes là face à une représentation dont le motif global ne surprend pas, mais dont l'interprétation des détails pose problème.

En ce qui concerne sa datation, celle proposée par T. Özgüç pour le niveau $3 b$ de la phase II, qu'il identifie dans son même article à la période Hittite ancienne ( $c a$. 1650-1500), correspond bien à celle que l'on attend pour ce type de vase. En effet, il semble désormais bien établi, notamment grâce aux dernières découvertes, que les jarres à quatre frises à reliefs datent de la période Hittite ancienne, période antérieure à l'évolution vers des schémas de composition plus libres sur d'autres formes de vases ${ }^{32}$. Quant au lieu de production du vase, aucun élément ne permet vraiment d'apporter des informations, faute d'analyse de pâte et à la vue de la répartition des parallèles convaincants. D'après la documentation, une attribution en Anatolie centrale, quelque part entre Bitik et Alişar est raisonnable, mais manque de précision.

26) Voir note 23.

27) von der Osten $1937: 111$, fig. 153-154.

28) Neve $1992: 31$, fig. 78 et Parzinger et Sanz $1992:$ Pl. 69, fig. 77.

29) Les six fragments de ce vase ne sont pas jointifs ; ils proviennent d'un locus précis et sont publiés à la manière d'une reconstitution de trois registres dont aucune information à ce sujet n'est fournie (Parzinger et Sanz $1992:$ Pl. 68, fig. 75). Les profils ne sont pas dessinés, ce qui empêche de vérifier s'il s'agit d'un autre jarre monochrome à quatre registres de reliefs.

30) Özgüç $1988: 86$.

31) Özgüç $1988: 175$, fig. 64.

32) Boehmer $1983: 21$; Seeher $2007: 715$. 


\section{A}

I

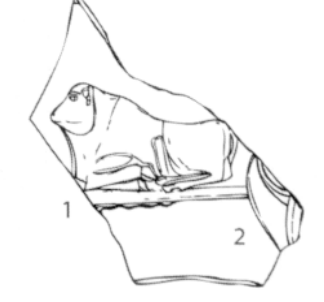

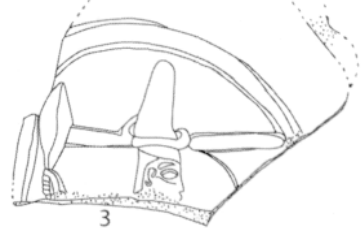

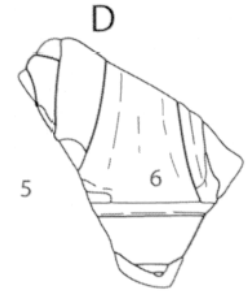

III

IV

E

$\mathrm{F}$

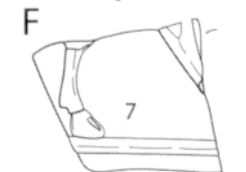

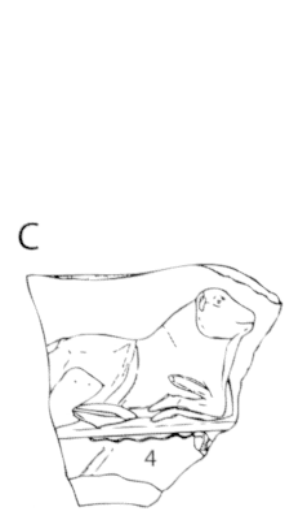
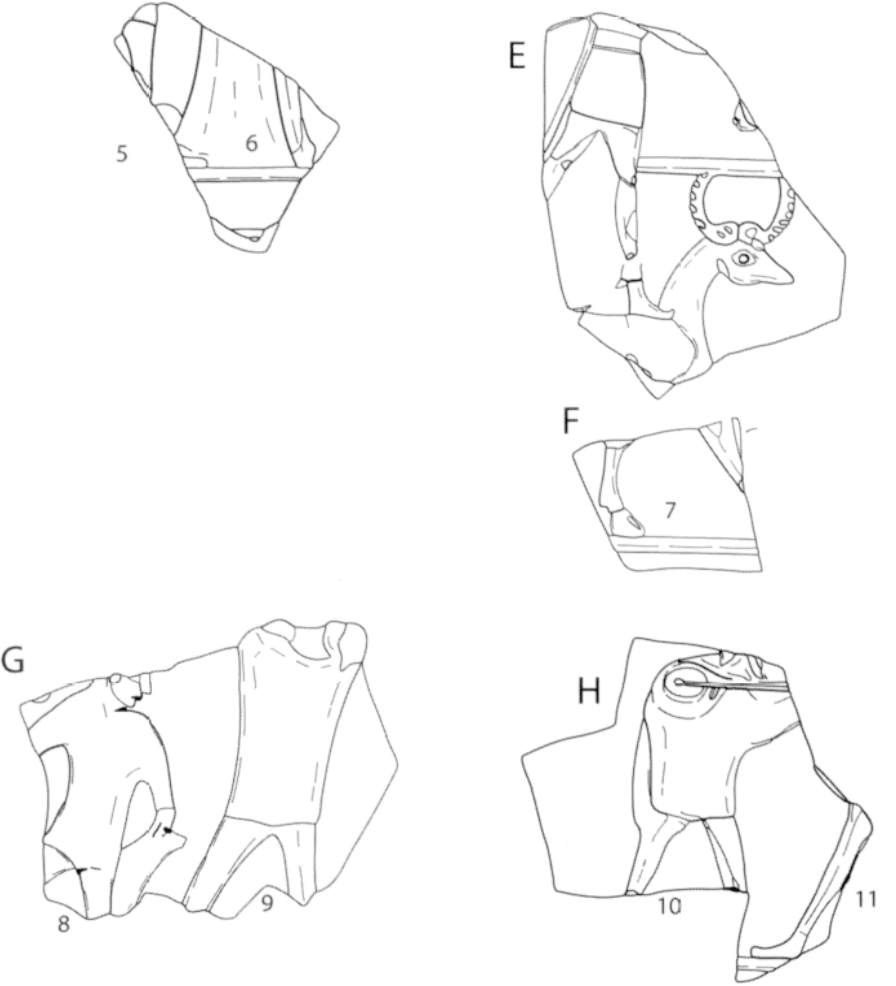
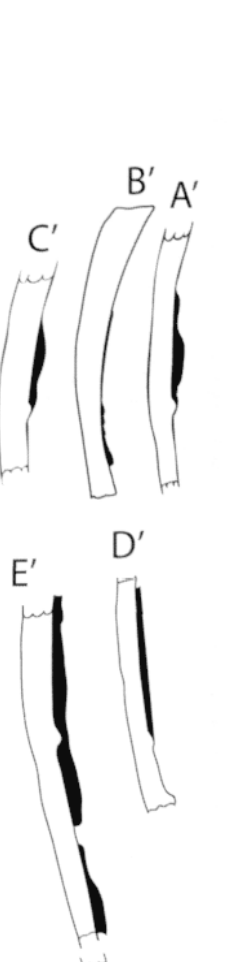

$F^{\prime}$

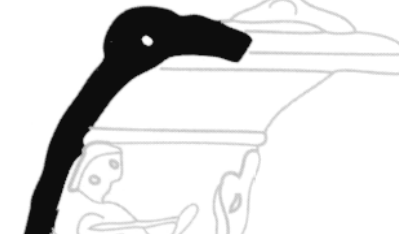



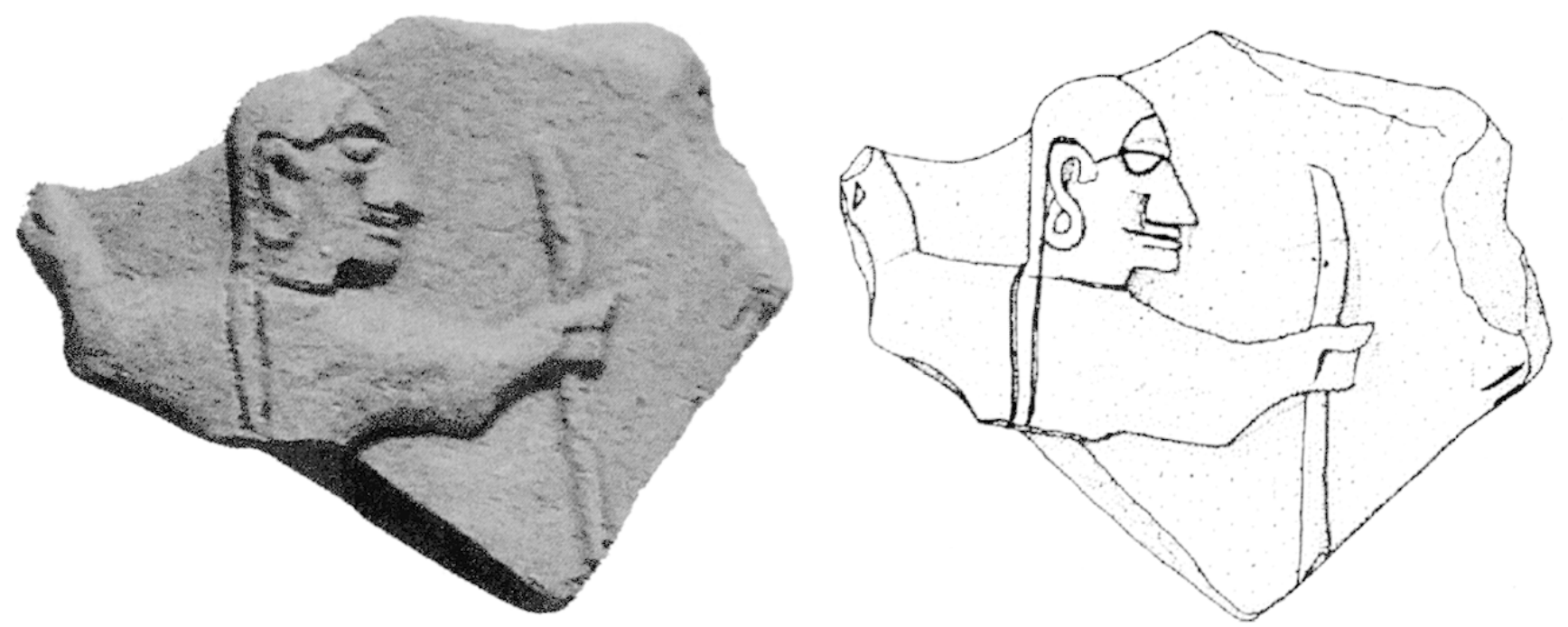

Fig. 5 : Fragment provenant de Boğazköy

(Photographie d'après Neve 1992: 31, fig. 78b et dessin d'après Parzinger et Sanz 1992 : pl. 67, fig. 64).

\section{LE VASE D'ESKIYAPAR}

Lors de la fouille d'Eskiyapar, différents fragments de cinq vases à reliefs furent découverts par $\mathrm{R}$. Temizer et publiés par T. Özgüç à la suite du vase d'İnandiktepe ${ }^{33}$. L'un de ces vases se distingue par sa pâte grise contenant du mica ce qui a permis de rassembler les tessons appartenant à ce vase sans difficulté ${ }^{34}$. Un fragment représentant la tête d'un dieu du même vase resta inédit jusqu'à sa publication avec deux statuettes de métal ${ }^{35}$. Selon son inventeur, la forme du vase est une jarre, ce qui nous a permis de proposer une reconstitution du programme décoratif en utilisant la même méthode que pour le vase d'Alaca Höyük : en créant des groupes de tessons ayant la même concavité et en les ordonnant par registre à l'aide des bandes en relief (Fig. 4). Là encore, la position d'aucun des tessons au sein d'un registre n'est assurée, plusieurs reconstitutions sont possibles en déplaçant horizontalement les fragments. La restitution présentée de ce vase est plus polémique que dans le cas du vase d'Alaca Höyük en raison des associations faites entre les différents registres et les difficultés d'interprétations rencontrées.

\subsection{L'iconographie du vase}

A l'évidence, le vase s'apparente à ceux qui possèdent quatre frises ornées d'une procession réunissant, entre autres, des musiciens. Trois fragments peuvent être placés sans difficultés au registre supérieur $(\mathrm{A}-\mathrm{C})$. Là, sont représentés deux bovins allongés, couchés sur la ligne d'appui, les pattes repliées sous le corps. Les quatre pattes illustrent un beau jeu de perspective où celle au premier plan est plus grosse. La queue passe sous les pattes et remonte sur le milieu du corps. L'encolure montre une dépression entre le corps et la tête soulignant le relief du taureau. La tête, entièrement de profil, est bien détaillée avec corne, oreille, œil et naseau. Comme on peut le retrouver sur un tesson stylistiquement très proche d'Alişar ${ }^{36}$, la frontalité est une des solutions choisies par les artistes hittites pour représenter les cornes même si le plus souvent les deux cornes étaient représentées de profil, encadrant la tête ${ }^{37}$.

Sous chaque taureau, de l'autre côté du bandeau en relief, court un motif en vaguelettes. Pour le taureau tourné à droite (4), le motif est interrompu

33) Özgüç $1988: 117-122$.

34) Les "quatre" autres vases à reliefs sont du type polychrome et sont stylistiquement similaires. Malheureusement tous les fragments ne sont pas dessinés, notamment deux tessons du "vase 1" (Özgüç 1988 : pl. 70, 3-4), et ils sont souvent de petite taille ce qui empêche une reconstitution solide du vase fondée sur les différences de courbure à partir des profils. En outre, la répartition des tessons en quatre vases s'appuie sur une différence estimée du diamètre des vases à partir des tessons, mais ne pourrait-on pas imputer cette variation de diamètre à la forme même du vase, en particulier dans le cas d'une jarre ? Pourrait-il s'agir, non pas de quatre vases mais d'un exemplaire unique ? Les fragments fig. 49-50:168-169 possèdent le même profil et exactement la même séquence décorative, mais appartiendraient à deux vases différents car l'un "est plus grand et plus lourd" que l'autre (Özgüç 1988: 119). Ou bien s'agirait-il d'une simple déformation provoquée par l'application des reliefs ? Une nouvelle étude des tessons pourrait, à notre avis, amener à la reconstitution d'un seul vase, plus complet que celui présenté ici.

35) Özgüç 1999.

36) von der Osten $1937: 116$, fig. $156=$ Bochmer $1983: 47$, fig. 38.

37) Par exemple à İnandıktepe, Yörüklü - Hüseyindede Tepesi pour la céramique à reliefs mais aussi dans d'autres arts figuratifs. A ce sujet voir les exemples relevés dans Yıldırım 2008 : 839-840. 
par un élément brisé dont la nature est inconnue. A la droite du taureau tourné sur la gauche (1), l'extrémité pointue d'une aile en faible relief (2) dépasse du deuxième registre et interrompt le motif en vaguelettes ainsi que la ligne d'appui. Cette aile s'unit à un bourrelet en haut-relief incurvé. T. Özgüç proposa de reconnaître un fragment de cercle et ainsi de reconstituer un disque ailé. La présence du bourrelet incurvé pourrait autrement indiquer le bras d'une divinité. Il faut admettre que la forme de l'aile est plus similaire à celle du dieu ailé conservé au Metropolitan Museum of Art de New-York ${ }^{38}$ qu'à l'image du disque solaire ailé que nous connaissons à travers la glyptique ou la sculpture monumentale uniquement à partir du XIV $\mathrm{XI}^{\mathrm{e}}$ siècle en Anatolie ${ }^{39}$.

Entre ces deux bovins nous avons placé le relief représentant la tête d'un dieu de profil, tourné sur la droite (3). Celui-ci est encadré, au minimum dans la partie supérieure, par une bande en relief arrondie qui tombe à la verticale derrière lui. Il est coiffé d'une tiare ovoïde munie d'une paire de cornes ce qui assure l'identification à un dieu. Le visage à la partie inférieure perdue est mis en valeur par l'œil surmonté d'un sourcil, la pommette saillante, le nez pointu et l'oreille en forme de S. La haute coiffe du dieu interrompt la bande supérieure du registre qui court horizontalement. Devant le dieu on distingue un méplat arrondi alors qu'il lève la main droite en arrière et saisit un objet oblong à rainure centrale qui scinde la face avant en deux pans. Malheureusement, la brisure sous le poignet rend la lecture et l'interprétation difficile. Il nous semble qu'un manche ou une hampe dépasse sous les doigts de la main, sans pouvoir préciser $s$ 'il est brisé ou non ${ }^{40}$. L'objet tenu dans sa main apparaît comme un unicum, il ne ressemble pas à un fer de lance, qui est typiquement représenté dans l'iconographie hittite par un triangle allongé ${ }^{4}$, et le soin général de la figure ne permet pas d'envisager une représentation fautive d'un objet si commun. De plus, une lance portée par la main arrière semble improbable ; dans ce cas il pourrait plutôt s'agir d'une masse d'arme (? ${ }^{42}$, ce qui serait en meilleure adéquation avec la position de la main (voir infra). La bande en relief qui encadre la tête de la divinité ne peut se comparer qu'à celle de la charrette dépassant la ligne du registre supérieur dans la jarre d'Yörüklü - Hüseyindede Tepesi ${ }^{43}$. S'agit-il, ici, de l'évocation d'une architecture devant laquelle se trouverait le dieu?

Sous ce tesson, nous avons placé en correspondance le grand fragment $E$ représentant un personnage monté sur un cerf, identifié avec la divinité reconnue dans l'écriture cunéiforme ${ }^{\mathrm{d}} \mathrm{KAL}$. La divinité debout, la jambe droite avancée, est court-vêtue et une longue mèche de cheveux en relief longe l'arrière du personnage. En association avec le cerf nous avons placé le tesson $F$ qui semble concorder avec l'enjambée du cerf ${ }^{74}$.

Seul un autre fragment peut être attribué au deuxième registre (D). On reconnait une longue jambe éffilée (5) de profil et dont le pied est situé en avant d'un objet convexe qui pourrait représenter un autel (6). Est-ce que la jambe svelte (5) pourrait être en lien avec "l'aile" (2)?

Enfin, le quatrième registre regroupe des musiciens dont un luthiste (10) qui fait face à une personne qui se dirige énergiquement à sa rencontre mais dont seule la jambe avant est conservée (11). Le luthiste porte un pagne court et un châle (?) que l'on devine entre le coude et la cuisse droite. A sa gauche et à sa droite, j'ai fait figurer le corps de personnages en vis-à-vis, qui ont les bras levés dans la posture typique des joueurs de cymbales/tambourin $(9,12)$. Le personnage tout à gauche (8), de plus petite taille, les épaules de face et les bras abaissés, saisi un objet de sa main gauche, difficilement identifiable.

\subsection{Parallèles}

Au niveau de la facture, ce vase monochrome s'inscrit bien dans le style des reliefs polychromes, et le modelé du dieu, très différent des visages du

38) "Winged deity [Anatolia] (1990.255)" in Heilbrunn Timeline of Art History. New York : The Metropolitan Museum of Art, 2000. http://www.metmuseum.org/toah/works-of-art/1990.255 (consulté en mai 2011).

39) Le disque solaire ailé est associé aux cartouches royaux depuis Šuppiluliuma I. Voir par exemple Boehmer et Güterbock $1987: 80$ et la classification dans Parayre 1984.

40) Insatisfait par le dessin publié par T. Özgüç (1999:38, fig. 1), j'ai redessiné le tesson à partir de la photographie fournie (Özgüç $1999: 38$, pl. 14). En effet, la bande en relief sur le côté gauche du tesson était totalement absente, la forme de l'objet tenu dans la main gauche trop ovoïde, l'extrémité dépassant sous le poignet n'était pas représentée. De même, le méplat en avant du dieu et les cornes de la tiare n'étaient pas fidèles. La partie supérieure du tesson en tireté, absente de la photographie, a été restituée en s'inspirant du dessin.

41) Parzinger et Sanz 1992 : pl. 67, fig. 65 ou encore Herbordt 2005 : Kat.169, 275-6, 504-7, 579, 629-30.

42) Voir les exemples dans Beyer 2001 : A.25 (les deux personnages de gauche) ou encore A.33, A.47, A.67.

43) Yildırım $2007: 850$, fig. 6.1.

44) T. Özgüç avait voulu reconnaître les membres d'un bovin et restituer un bovin debout, attribut du dieu de l'orage. Il semble cependant, à l'unique vue du sabot et du peu de pattes conservées, qu'il ne soit pas évident de faire la distinction entre animaux au sabot fourchu. 


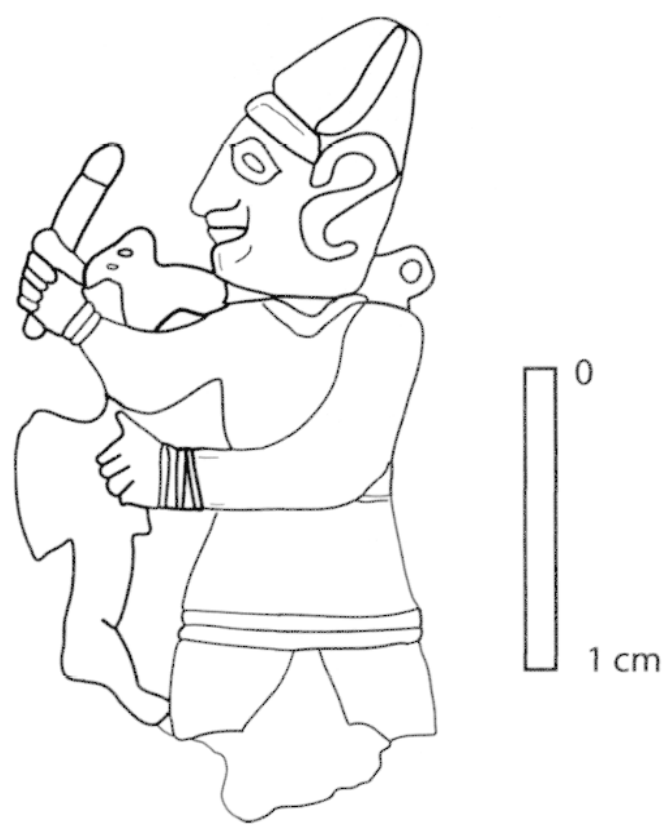

Fig. 6 : Pendentif en lapis-lazuli représentant le dieu ${ }^{\mathrm{d}}$ KAL, découvert à Aššur. Dessin exécuté d'après photographie (Andrae 1924 : pl. 8f).

vase d'Alaca Höyük, souligne que le style de ce vase est beaucoup plus proche de la tradition des vases polychromes : il correspond aux stéréotypes connus.

Issu de la céramique, seul un relief monochrome peut être comparé à la reconstitution de la divinité ${ }^{d} \mathrm{KAL}$ (Fig. 5). Il s'agit d'un personnage à la mèche de cheveux dépassant sur l'épaule, il lève le bras en arrière mais sa main est perdue ce qui nous empêche de comparer l'objet saisi ; il tient un élément rectiligne dans la main gauche, hampe ou bride (?) mais ne porte pas de tiare à cornes.

Dès lors, il est indispensable de s'interroger sur les différentes représentations de la divinité que nous connaissons pour élargir le champ des comparaisons. L'écriture cunéiforme ${ }^{\mathrm{d}} \mathrm{KAL}$, qui peut être augmentée de compléments phonétiques, recouvre tout un ensemble de divinités protectrices, que l'on connait nominalement (ex. Inara, Kurunta, Lullayama) ou qui sont attestées pour des institutions (ex. le palais, le temple) ; il existe également des divinités protectrices personnelles ${ }^{45}$. C'est grâce aux textes bien connus des descriptions d'images divines que le dieu perché sur un cerf a été identifié avec la divinité ${ }^{d} \mathrm{KAL}$. La description notoire du dieu protecteur de la ville de Wiyanawanta est explicite à ce sujet : "dKAL.LÍL. Idole : une statuette d'homme en or, debout, [casqué] ${ }^{46}$. Dans la main droite il tient un arc en or ; dans la main gauche il tient un aigle d'or (et) un lièvre d'or, un poignard d'or - dessus des fruits d'or. Il se dresse sur un cerf debout"47. Cette description a été associée à différentes représentations où le plus souvent le lituus remplace l'arc comme sur la petite plaquette en stéatite de Yeniköy ${ }^{48}$; le rython de la collection Norbert Schimmel ${ }^{49}$ ou le pendentif en argent découvert à Chypre ${ }^{50}$. On retrouve le dieu 'KAL représenté masse d'arme à l'épaule sur un sceau des archives de Nişantepe ${ }^{51}$ ou arc à l'épaule sur la stèle d'Altınyayla ${ }^{52}$. L'oiseau est un attribut de la divinité, qui n'est pas toujours présent. Les études précédentes ont montré que ces représentations distinctes devaient symboliser différentes divinités protectrices ${ }^{53}$. L'adéquation bien établie entre le cunéiforme ${ }^{\mathrm{d}} \mathrm{KAL}$ et le hiéroglyphique (DEUS)CERVUS ${ }^{54}$ insiste sur la symbolique du cerf, un des attributs des divinités protectrices, dont la continuité entre l'époque des Comptoirs de Cappadoce et le Royaume hittite semble évidente ${ }^{55}$.

Sous une iconographie légèrement différente, on reconnait le dieu protecteur ${ }^{\mathrm{d}} \mathrm{KAL}$ dans un personnage en lapis-lazuli d'Aššur (Fig. 6). Sous un aspect de chasseur, il tient un oiseau, un lapin et un poignard/masse d'arme(?) ; von der Osten-Sacken pensait à un lituus $^{56}$. L'objet ressemble à celui tenu par le dieu ${ }^{\mathrm{d}} \mathrm{KAL}$ dans ma restitution du vase d'Eskiyapar ${ }^{57}$.

45) Haas $1994: 449-451$.

46) Pour "casqué" voir Haas $1994: 498$.

47) D'après Laroche 1983.

48) Bittel 1976 : fig. 247.

49) Muscarella $1974: 123$.

50) Christou 1993 : 734, fig. 36 ; South 1997 : pl. XV, fig. 1.

51) Herbordt $2005:$ Kat. 186.

52) Müller-Karpe 2003.

53) Par exemple : Güterbock 1983, von der Osten-Sacken 1988 ; McMahon 1991 ; Haas 1994 : 449-459 ; Herbordt 2005 : 60-62 ; Hawkins 2006 ; Haas $1994: 451$ "Schutzgottheiten existieren in beinahe unbeschränkter Anzahl".

54) Pour une courte synthèse : Hawkins $2005: 290$.

55) Mouton 2002 : 95-96.

56) von der Osten-Sacken $1988: 65$, note 9.

57) Très similaire, on peut comparer le relief du dieu de l'Orage du temple d'Alep : Kohlmeyer et al. (2005). Il faut souligner que l'interprétation d'attributs, même intacts, est loin d'être évidente ; l'objet tenu dans la main gauche par une déesse sur le treizième bloc du temple en est un bon exemple Kohlmeyer et al. (2005). 


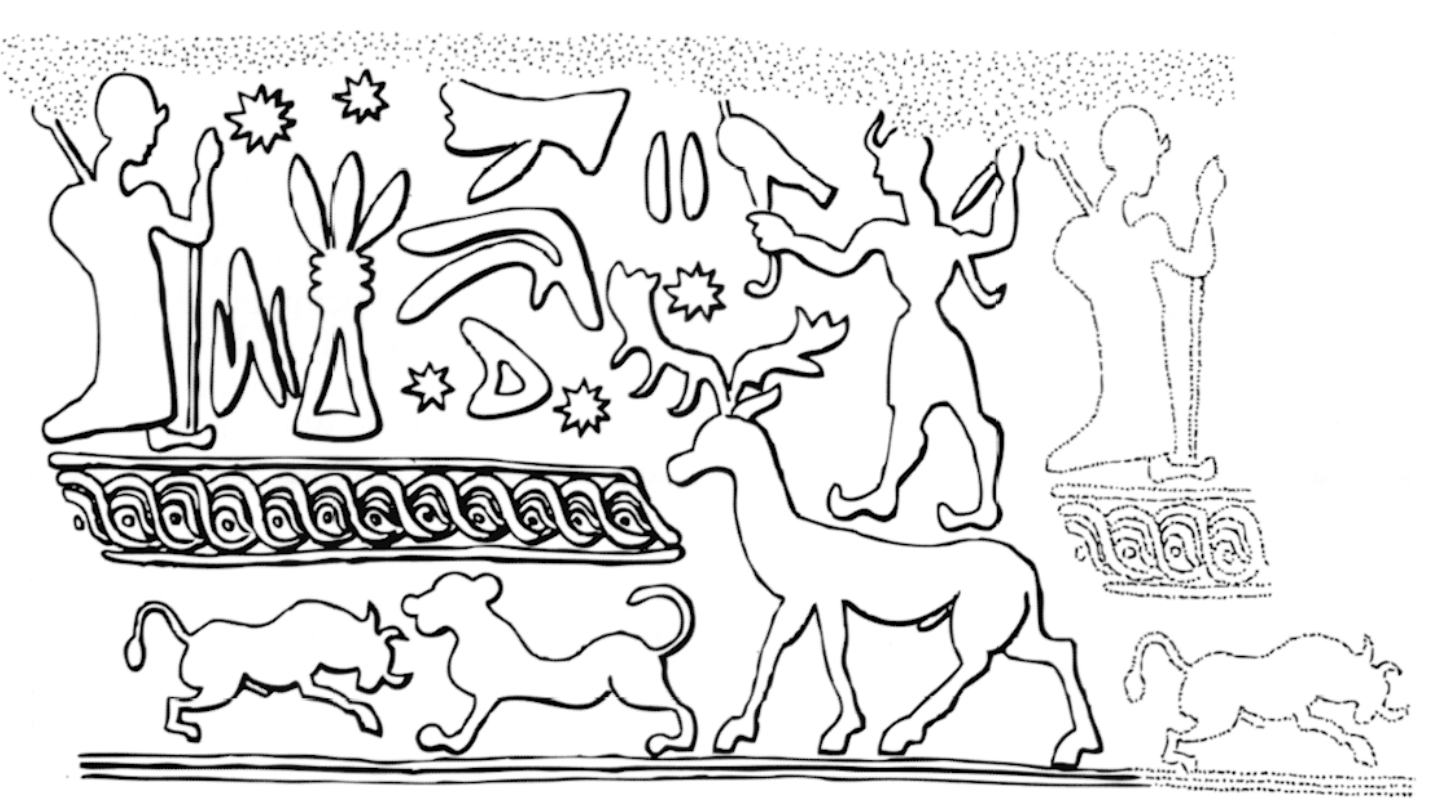

Fig. 7 : Empreinte A78 d'un sceau-cylindre découvert sur une tablette cunéiforme d'Emar (Beyer 2001 : 94, A.78).

Dans la restitution proposée, une identification à une divinité ${ }^{\mathrm{d}} \mathrm{KAL}$, grâce à la présence du cerf ne pose aucune difficulté et l'arme tenue se rapproche de celle du pendentif d'Aššur. La position arrière du bras trouve écho dans l'empreinte d'un sceau syroanatolien d'Emar (plus tardif) où le dieu 'KAL, perché sur un cerf, tient une masse d'arme dans sa main levée vers l'arrière (Fig. 7) ${ }^{58}$. La comparaison est particulièrement intéressante puisqu'elle ne s'arrête pas à la posture du dieu mais également à l'utilisation du champ de la représentation. En effet, le programme iconographique du cylindre A78 se développe grâce à un "double registre partiel, cette formule étant certainement à mettre à l'actif d'influences syrienne et mitanienne" 59 . Cette organisation est un parallèle exact à celle du vase d'Eskiyapar où le Dieu au cerf est représenté sur deux registres et l'empreinte déroulée sur la tablette ME 13 place le dieu exactement en son milieu ${ }^{60}$. Lors de son commentaire sur le groupe des cylindres syro-hittites, Beyer établit "l'empreinte A78 présente une disposition tout à fait originale : au registre supérieur un orant royal est placé face au groupe des hiéroglyphes du nom du propriétaire. A l'autre extrémité, occupant toute la hauteur du champ, la figure du dieu au cerf est ainsi mise en valeur" ${ }^{\prime \prime}$. Cette indication est très précieuse car la représentation d'une divinité sur deux registres est, pour la céramique à reliefs, également extraordinaire. Même si les sceaux d'Emar sont datés du XIV ${ }^{e}$ siècle, n'y a t-il pas un jeu d'influences similaire? Non seulement la position de la divinité, mais aussi l'ordonnance en deux registres correspondraient à des influences méridionales. On remarque d'ailleurs, dans le cas de la jarre représentant le dieu de l'Orage d'Alep ${ }^{62}$, que J. Seeher a envisagé une origine syrienne du motif.

\subsection{Ordonnance}

La place de cette œuvre au sein des vases à reliefs n'est pas aisée à déterminer. La forme de la jarre, le style des personnages, le motif et la division en quatre registres nous indiquent que le vase appartient à la période Hittite ancienne (Fig. 8). Encore peut-on suggérer une production plus tardive que les vases d'Inandıktepe et de Yörüklü - Hüseyindede Tepesi, par l'abandon de la représentation strictement divisée en registres et par la représentation d'un personnage sur deux registres, tendance qui aboutira à la disparition complète des registres selon le modèle $\operatorname{admis}^{63}$. De très bonnes questions ont été soulevées par J. Seeher, lorsqu'il s'interroge sur le

58) Beyer 2001 : A. 78 ; voir également : 330-331.

59) Beyer $2001: 32$.

60) Beyer $2001:$ pl. 40c

61) Beyer $2001: 35$.

62) Seeher $2007: 715$.

63) Boehmer 1983:21; Seeher $2007: 715$. 


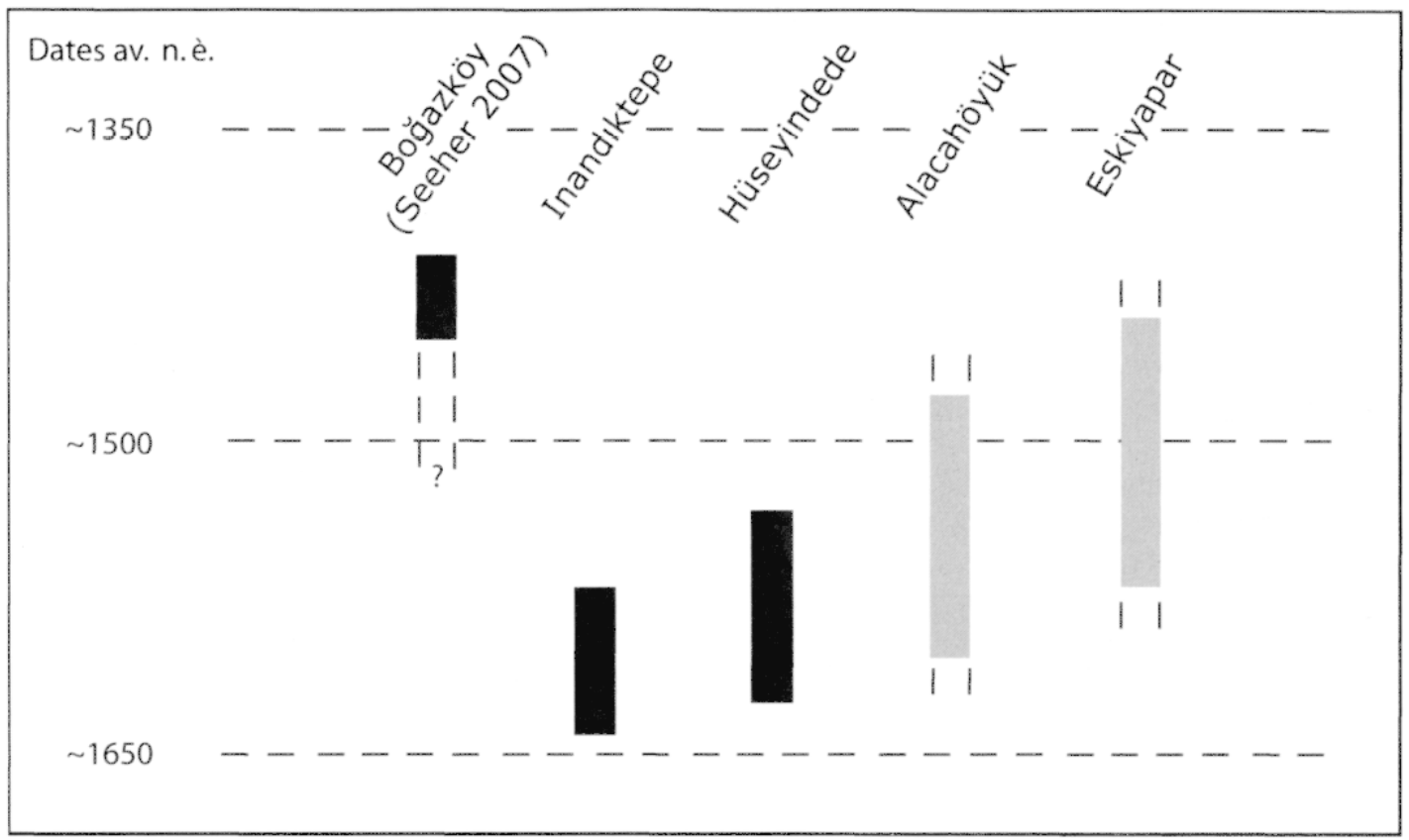

Fig. 8 : Tableau chronologique situant les trois vases à reliefs bien datés et les reconstitutions proposées. Plus le rectangle est allongé, moins la datation est précise.

lieu et l'origine du vase ou de l'artiste qui a produit la frise illustrant des sphinx et le dieu de l'Orage d'Alep embarqué sur son char tiré par des bœufs. Pour le vase d'Eskiyapar la pâte micacée implique une pâte différente de celle des autres vases découverts à Eskiyapar ${ }^{64}$ et atteste sans aucun doute d'une origine différente des vases - ce qui permet de relever un phénomène d'échanges, largement supposé pour les vases à reliefs mais attesté pour la première fois. Quant au lieu de production, on peut proposer à titre d'hypothèse, et si l'on se fonde sur l'iconographie, une attribution aux régions méridionales du Royaume, ou du moins que l'artisan ait baigné dans ce milieu.

\section{CONCLUSION}

La reconsidération globale de ces deux vases a apporté de nombreux éléments qui n'avaient pas suffisamment été exploités lors des différentes publications. Ainsi, deux "nouveaux" vases à reliefs complètent une documentation toujours grandissante. Il est désormais certain que la production de vases monochromes accompagne le floruit de la production de jarres à reliefs polychromes pendant la période de l'ancien Royaume. Cette similitude montre à quel point la production des ces jarres à quatre reliefs est uniforme : elles sont retrouvées sur de nombreux sites de la période Hittite ancienne. Tout comme d'autres innovations architecturales et techniques, sans doute en partie autochtones ${ }^{65}$, la conception des vases à reliefs à quatre frises, dont le schéma de base est formellement respecté semble s'être répandue rapidement au sein de l'ancien Royaume hittite. Sans aucun doute, les vases à reliefs, aux caractéristiques techniques attestant d'une production locale, sont des marqueurs d'une identité culturelle forte dont les artisans, si nombreux aient-ils été, en maitrisaient parfaitement la grammaire. Il en découle que les vases à reliefs témoignent de l'uniformisation d'objets rituels dès la formation du Royaume hittite.

N.S.

\section{REFERENCES}

Andrae, W., 1924 : Hethitische Inschriften auf Bleistreifen aus Assur. Hinrichs, Leipzig.

Arık, R. O., 1937 : Les fouilles d'Alaca Höyük entreprises par la société d'histoire turque. Rapport préliminaire sur les travaux en 1935. Türk Tarih Kurumu Basım Evi.

Arnhold, S., 2009 : Das hethitische Gebäude E auf der Akropolis von Kuşakll. Kuşaklı-Sarissa IV. Marie Leidorf, Rahden.

Baudry, M.-T. (éd.), 1978 : La sculpture : méthode et vocabulaire. Imprimerie Nationale, Paris.

64) Sur "les vases" découverts à Eskiyapar, voir la note 34.

65) Schachner 2009a: 15-18. 
Beyer, D., 2001 : Emar IV - Les Sceaux. Vandenhoeck \& Ruprecht, Fribourg.

Bittel, K., 1955 : "Eine hethitische Reliefvase aus Kappadokien", in Bruns, G. (éd.), Festschrift für Carl Weickert, Berlin, 23-33.

- 1976 : Les Hittites. Gallimard, Paris.

Boehmer, R. M., 1983 : Die Reliefkeramik von Boğazköy ; Grabungskampagnen 1906-1912, 1931-1939, 1952 - 1978, Boğazköy-Hattuša XIII. Mann, Berlin.

Boehmer, R. M. et Güterbock, H. G., 1987 : Glyptik aus dem Stadtgebiet von Boğazköy, Boğazköy-Hattuša XIV. Mann, Berlin.

Christou, D., 1993 : "Chronique des fouilles et découvertes archéologiques à Chypre en 1992", Bulletin de Correspondance Hellénique 117 : 719-755.

Güterbock, H.-G., 1983 : "Hethitische Götterbilder und Kultobjekte", in Boehmer, R. M. et Hauptmann, H. (éds.), Beiträge zur Altertumskunde Kleinasiens, Festschrift für Kurt Bittel, Mainz am Rhein : 203-217.

Haas, V., 1994 : Geschichte der hethitischen Religion. Brill, Leiden.

Hawkins, J. D., 2005 : "Commentaries on the readings", in Herbordt 2005 : 250-253.

- 2006 : "Tudhaliya the hunter", in Hout, Th. P. J. van den. (éds.), The Life and Times of Hattušili III and Tuthaliya $I V$, Nederlands Instituut voor het Nabije Oosten, Leiden : 49-76.

Herbordt, S., 2005 : Die Prinzen- und Beamtensiegel der hethitischen Grossreichszeit auf Tonbullen aus dem Nişantepe-Archiv in Hattuša. Boğazköy-Hattuša XIX. von Zabern, Mainz am Rhein.

Kohlmeyer, K., Gonnella, J. et Khayyata, W., 2005 : Die Zitadelle von Aleppo und der Tempel des Wettergottes. Rhema, Münster.

Koşay, H. Z., 1951 : Alaca Höyük Kazısı 1937-1939 daki Çalışmalara ve Keşiflere Ait İk Rapor - Les fouilles d'Alaca Höyük entreprises par la Société d'Histoire Turque, rapport préliminaire sur les travaux en 19371939, Türk Tarih Kurumu Basım Evi, Ankara.

Laroche, E., 1980-1983 : "Lamma/Lamassu in Anatolien", Reallexikon der Assyriologie, $6: 455-459$.

McMahon, G., 1991 : The Hittite State Cult of the Tutelary Deities, Oriental Institute of the University of Chicago, Chicago.

Mielke, D. P., 2006 : Die Keramik vom Westhang. Kuşakl1-Sarissa II, Marie Leidorf, Rahden.

Müller-Karpe, A., 2003 : "Die Stele von Altınyayla ein neues Relief der hethitischen Grossreichzeit", in Özdoğan, M. (éd.), Köyden kente, Arkeoloji ve Sanat Yayınları, Istanbul : 313-319. Mainz.

Muscarella, O. W., 1974 : Ancient art, von Zabern,

Mouton, A., 2002 : "Y a-t-il une relation entre les motifs de la glyptique cappadocienne et les hiéroglyphes anatoliens ?", Studi Micenei Ed Egeo-Anatolici XLIV : 83-113.
Neve, P., 1992 : Hattuša-Stadt der Götter und Tempel, von Zabern, Mainz am Rhein.

von der Osten, H. H., 1937 : The Alishar Höyük, Seasons of 1930-32 Part II, Univ. of Chicago Press, Chicago.

von der Osten-Sacken, E., 1988 : “Der kleinasiatische Gott der Wildflur", Istanbuler Mitteilungen, 38 : 63-81.

Özgüç, T., 1957 : “The Bitik Vase”, Anadolu / Anatolia, revue annuelle d'archéologie II : 57-78.

- 1982 : Maşat Höyük II, Boğazköy'ün Kuzeydoğusunda Bir Hitit Merkezi ; A Hittite Center Northeast from Boğazköy, Türk Tarih Kurumu Basım Evi, Ankara.

- 1988 : Inandiktepe, Eski Hitit Çağında Önemli Bir Kült Merkezi ; An Important Cult Center in the Old Hittite Period, Türk Tarih Kurumu Basım Evi, Ankara.

- 1993 : "Studies on Hittite Relief Vases, Seals, Figures and Rock-Carving", in Mellink, M. J., Porada, E., et Özgüç, T. (éds.), Aspects of Art and lconography: Anatolia and its Neighbors. Studies in Honour of Nimet Özgüç, Ankara : 473-499.

- 1999 : "The Metal Figurines of two Hittites Gods", Bulletin of the Middle eastern Cultur Center in Japan XI - Essays on Ancient Anatolia : 23-38.

Parayre, D., 1984 : "Nouvelle approche de la glyptique 'mitannienne' : le disque ailé de Kirkuk à Tyrinthe et d'Alishar à Meskene", in Barrelet, M.-T. (éd.), Problèmes concernant les Hurrites II, Ed. Recherches sur les Civilisations, Paris : 213-260.

Parzinger, H. et Sanz, R., 1992 : Die Oberstadt von Hattuša ; Hethitische Keramik aus dem zentralen Tempelviertel, Boğazköy-Hattuša XV. Mann, Berlin.

Schachner, A., 2009a : "Das 16. Jahrhunderts v. Chr - eine Zeitenwende im hethitischen Zentralanatolien", Istanbuler Mitteilungen 59 : 9-34.

- 2009b : "Die Ausgrabungen in Boğazköy-Hattuša 2008", Archäologischer Anzeiger 1 : 21-72.

Seeher, J., 2007 : "Eine Kultvase mit der Darstellung des Wettergottes von Halab aus Hattuša", in Alparslan, M., Doğam-Alparslan, M., et Peker, H. (éds.), Belkıs Dinçol ve Ali Dinçol'a Armağan VITA - Festschrift in Honour of Belkıs Dinçol and Ali Dinçol, İstanbul : 707720 .

Sipahi, T., 2000 : "Eine althethitische Reliefvase vom Hüseyindede Tepesi", Istanbuler Mitteilungen 50 : 63-85.

- 2005 : "Hüseyindede Tepesinde Bulunan Yeni Bir Kült Vazosu", in Süel, A. (éd.), Acts of the $V^{\text {th }}$ International Congress of Hittitology, Nokta Ofset, Ankara : 761-78.

South, A. K., 1997 : “Kalavasos-Ayios Dhimitrios 1992-1996", Report of the Departement of Antiquities of Cyprus : 151-175.

Yildırım, T., 2008 : "New scenes on the second relief vase from hüseyindede and their interpretation in the light oh the hittite representatier art", in Archi, A. et Francia, R. (éds.), VI Congresso Internazionale di Ittitologia, volume II : 837-850. 